\title{
Synthesis and compatibilization ability of hydrogenated polybutadiene- $b$ - polyamide 6 diblock copolymer in low density polyethylene and polyamide 6 blends
}

\author{
C. Harrats ${ }^{\mathrm{a}}, \mathrm{R}$. Fayt ${ }^{\mathrm{b}}$ and R. Jérôme ${ }^{\mathrm{b}}$ \\ ${ }^{a}$ Laboratory for Macromolecular Structural Chemistry, Department of Chemistry, Katholieke \\ Universiteit Leuven, Celestijnenlaan, 200 F, 3001, Heverlee, Belgium \\ ${ }^{\mathrm{b}}$ Center for Education and Research on Macromolecules, University of Liège, B6 de Chimie, \\ Sart-Tilman, 4000, Liège, Belgium
}

\begin{abstract}
This paper describes the synthesis of hydrogenated polybutadiene- $b$-polyamide 6 (HPB- $b$ PA6), a pure diblock copolymer, which consists of three main steps: synthesis of hydroxyl terminated polybutadiene, its hydrogenation and functionalization with an $\mathbf{t}$-caprolactam blocked diisocyanate. In a third step the functionalized HPB is copolymerized with $\mathbf{E}-$ caprolactam monomer via ring opening anionic polymerization.
\end{abstract}

The compatibilization ability of the synthesized pure diblock copolymer was evaluated in low-density polyethylene (LDPE)/polyamide 6 (PA6) blends. The investigation includes phase morphology development using both optical and electron scanning microscopy. The ultimate tensile properties of the modified and non-modified blends were also evaluated. The synthesized pure diblock copolymer exhibits very interesting interfacial activity both in terms of particle size reduction and improvement of the interfacial adhesion between the incompatible LDPE/PA6 phases. The tensile properties of the investigated blends were also significantly affected by the addition of the copolymer. The efficiency of compatibilization was found to be very sensitive to the molecular characteristics such as composition and molecular weight of the copolymer. Among the copolymers investigated a copolymer containing $24 \mathrm{wt} \%$ PA6 and having a total molecular weight $\bar{M}_{\llcorner\mathrm{of}} 87,000$ was found to exhibit the most efficient interfacial activity.

Keywords: Polyamide; Low density polyethylene; Compatibilization

\section{Introduction}

Blending of polyamide and polyolefin homopolymer pair is widely reported in blend literature. Their compatibilization is carried out mainly via reactive processing. The technique consists of using an intermediate reactive copolymer, which is physically compatible with the polyolefin phase and is able to chemically react with polyamide homopolymer to in situ form a compatibilizing copolymer. The reactive approach, although wide spread, still suffers from a lack of control and characterization. Nevertheless, many reports dealt with the investigation of the inter-relation between the interfacial grafting reaction, the phase morphology developed and the ultimate properties of the designed blend [1-6]. Industrials are challenged by the design of high-performance equipment (multistage-extruders and associated screws, batch mixers, roll mill, etc.) for the realization of efficient reactive processing operations. Scientists and polymer chemists are facing the most demanding problem of the molecular design of new functional polymers of well controlled and known molecular structure that should be able to efficiently react, under the processing conditions (temperature, pressure, residence time, shear, etc.), to promote in situ an efficient compatibilizer [7-10]. Ethylene-glycidyl 
methacrylate (E-GMA) and ethylene-acrylic acid (EAA), or maleic anhydride modified polyolefins are the most frequently employed reactive intermediates in polyolefin/PA blends. Nowadays maleic anhydride graft copolymer is progressively being less desired in applications where health concern is prevailing. Their substitution by 'eco-friendly' copolymer is expected to be neither economically nor technically simple. Anhydride groups react very efficiently in the melt with amine or hydroxyl bearing polymers to form, in situ, a graft copolymer at the interface between the immiscible polymer pair. To our knowledge, the use of pre-made HPB- $b$-PA6 pure diblock copolymer as an alternative to the reactive approach has never been reported so far for polyethylene/polyamide 6 blend compatibilization.

\section{Experimental}

\subsection{Materials}

Homopolymers. The homopolymers used are a low-density polyethylene Shell 33 grade from Shell (MFI $=0.3$, DENSITY $=0.922 \mathrm{~g} / \mathrm{cm}^{3}, \bar{M}_{\mathrm{L}}=40,000$ ) and a polyamide 6 Ultramid B4 from BASF (density $=1.08 \mathrm{~g} / \mathrm{cm}^{3}$ ) having an average molecular weight $\overline{\boldsymbol{M}}_{\mathrm{Lof}} 20,000$.

\subsection{HPB-b-PA6 synthesis}

Synthesis of hydroxyl terminated hydrogenated polybutadiene. Polybutadiene block was prepared via anionic polymerization of 1,3 butadiene monomer in toluene using sec-Butyl Lithium $\left(s\right.$-BuLi) initiator for $12 \mathrm{~h}$ at a polymerization temperature of $30^{\circ} \mathrm{C}$ [11]. Ethylene oxide monomer, purified on $n$-BuLi, was then added to the solution of living $\mathrm{PB} \mathrm{Li}^{+}$at a temperature of $0{ }^{\circ} \mathrm{C}$. The end-capping reaction was allowed to proceed for $1 \mathrm{~h}$. To hydrolyze the formed lithium alcoolate $\left(\mathrm{PB}-\mathrm{O}^{-} \mathrm{Li}^{+}\right)$living species few drops of a hydrochloric $\mathrm{acid} / \mathrm{methanol} \mathrm{mixture} \mathrm{were} \mathrm{added} \mathrm{to} \mathrm{the} \mathrm{solution.} \mathrm{The} \mathrm{complete} \mathrm{hydrogenation} \mathrm{of} \mathrm{the}$ hydroxyl terminated polybutadiene was performed according to Falk method using a $\mathrm{Co}^{2+} / \mathrm{Al}^{3+}$ Ziegler Natta catalyst under mild conditions $\left(\mathrm{H}_{2}: 50\right.$ Psi, $\left.T^{0}=60{ }^{\circ} \mathrm{C}, 1 \mathrm{~h}\right)[12]$.

Preparation of an acyllactam derivative. The hydroxyl terminated hydrogenated polybutadiene (HPB-OH), a suitable amount of $\epsilon$-caprolactam (needed for the formation in situ of sodium lactamate catalyst) and $\mathrm{t}$-caprolactam-blocked isocyanate (a molar excess of five times with respect to HPB-OH) were dissolved in a 1/10 decahydronaphtalene $(\mathrm{DHN}) /$ toluene mixture. After complete toluene distillation, which simultaneously eliminates any traces of water, the temperature was increased and stabilized at $105^{\circ} \mathrm{C}$. At this temperature, the reaction was initiated by the addition of sodium hydride [13]. After $1 \mathrm{~h}$ of reaction the polymer was recovered by precipitation in methanol, filtered and washed at least three times with methanol to eliminate the excess of e-caprolactam blocked-diisocyanate. The obtained polymer was characterized by FTIR which clearly reveals absorption bands at 1545 , 1637 and $3289 \mathrm{~cm}^{-1}$ characteristics of combined $\mathrm{NH}$ deformation and $\mathrm{CN}$ stretch, $\mathrm{C}=\mathrm{O}$ (amide) and NH stretch, respectively, (Fig. 1(a) and (b)). The chemical reaction scheme which illustrates the acyllactam formation is presented in Fig. 2. 


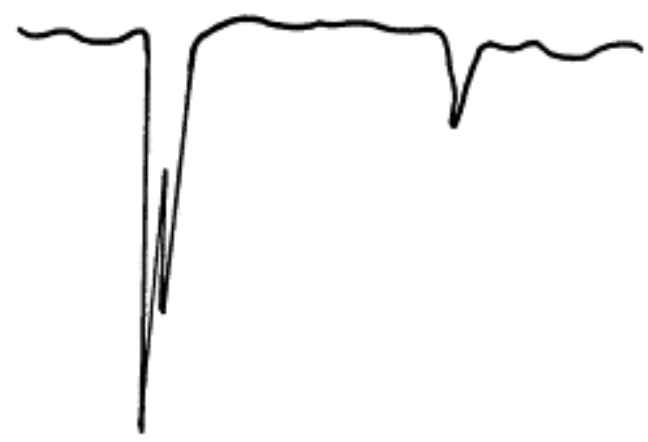

\begin{tabular}{ccccccccccc}
\hline 1 & 1 & 1 & 1 & 1 & 1 & 1 & 1 & 1 & \\
4000 & 3600 & 3200 & 2800 & 2400 & 2000 & 1600 & 1200 & 800 & 400
\end{tabular}
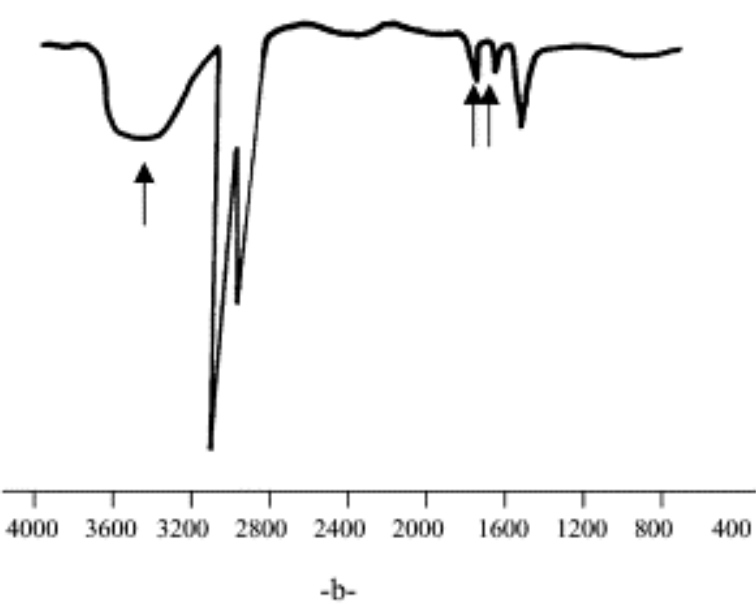

Fig. 1. FTIR spectrum of hydrogenated polybutadiene (a) before and (b) after isocyanate functionalization.

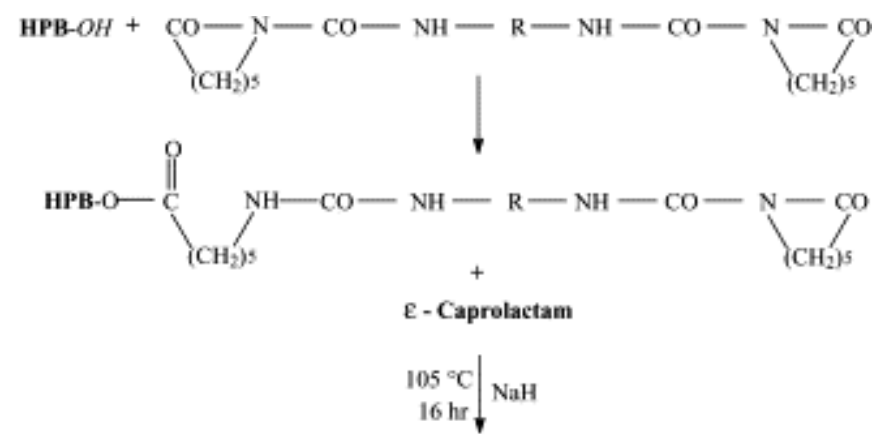

HPB-b-PA6

Fig. 2. Acyllactam formation and $\mathbf{\epsilon}$-caprolactam copolymerization.

Block copolymerization of functional HPB with E-caprolactam. In a typical experiment, $10 \mathrm{~g}$ of functional HPB and $10 \mathrm{~g}$ of dried $\mathrm{E}$-caprolactam were dissolved in 1/10 DHN/toluene mixture. After complete toluene removal by azeotropic distillation, the reaction temperature was set at $105^{\circ} \mathrm{C}$. Equimolar quantity of sodium hydride with respect to functional HPB was added to the reaction medium under nitrogen flow. The reaction was allowed to proceed for $16 \mathrm{~h}$. At the end of the polymerization, the crude insoluble polymeric product precipitated from the reaction medium. The copolymer was purified by successive extractions of the crude 
product as follows: (a) methanol extraction, in order to remove the non-reacted $\mathbf{t}$-caprolactam monomer; (b) hot toluene was used for removal of hydrogenated polybutadiene homopolymer; and (c) the homopolyamide (polycaprolactam) was extracted by using trifluoroethanol.

Because of the insolubility of HPB- $b$-PA6 the determination of its molecular weight was based on the molecular weight of the polybutadiene using classical SEC characterization and on the weight fraction of the PA6 block derived from successive extraction experiment. The molecular characteristics of the synthesized HPB- $b$-PA6 diblock copolymers, C1, C2 and C3 are gathered in Table 1.

Table 1. Molecular characteristics of HPB-b-PA6 diblock copolymer

\begin{tabular}{lllccc}
\hline Copolymer reference & $M_{a}$ HPB- $b$-PA6 & $M_{a}$ HPB & $M_{w} / M_{a}$ HPB & Wis PA6 & Copolymer comversion $\%^{\mathrm{a}}$ \\
\hline $\mathrm{C} 1$ & 12,500 & 10,000 & 1.01 & 20 & 75 \\
$\mathrm{C} 2$ & 64,000 & 50,000 & 1.03 & 22 & 75 \\
$\mathrm{C} 3$ & 87,000 & 66,000 & 1.09 & 24 & 65 \\
\hline
\end{tabular}

Blend preparation. 80/20 and 20/80 wt/wt LDPE/PA6 blends were prepared using a laboratory internal mixer (Brabender model) under nitrogen flow at a mixing temperature of $240{ }^{\circ} \mathrm{C}$. The copolymer $(10 \mathrm{wt} \%$ with respect to the total weight of LDPE+PA6) was melt milled with the minor phase prior to the addition of the major component. After mixing for $5 \mathrm{~min}$ at blades speed of $30 \mathrm{rpm}$, the milled mass was compression molded at $240{ }^{\circ} \mathrm{C}$ for $3 \mathrm{~min}$. The molded sheets were then rapidly cooled down to room temperature in a cold press. Tensile test specimens were cut according to DIN 53448 from the so-molded sheets. Each tensile value is the average of five tests performed on a Adhamel Lhomargy tensile tester at room temperature and at a cross-head speed of $20 \mathrm{~mm} / \mathrm{min}$.

Phase morphology of the blend was examined by using both SEM and optical microscopy. To determine the size of the dispersed PA6 particles in LDPE matrix, cryo-smoothed surfaces were etched by using trifluoroethanol, a selective solvent for PA6. The particle size measurement was carried out using a computer-added image analysis support on SEM micrographs.

The co-continuous phase morphology developed in the 20LDPE/80PA6 blend was examined by optical microscopy on microtomed sections of $1 \mu \mathrm{m}$ thick. Extraction of the major PA6 phase using trifluoroethanol was carried out on these blends in order to have a quantitative measure of the percentage of phase co-continuity. The percentage of LDPE phase continuity in PA6 matrix is determined from the weight of the biggest bulky LDPE particle, recovered after extraction of all PA6, with respect to the total weight of LDPE initially contained in the blend (20 wt \%). This achieved also additional extraction of the LDPE phase using hot dichloroethylene, its separation from the insoluble copolymer and the subsequent weighing of the recovered PA6 was necessary.

\section{Results and discussion}

\subsection{HPB-b-PA6 synthesis}

The synthesis of PB- $b$-PA6 was investigated earlier in our laboratory using different initiators (also defined as activators) such as ester, acid chloride and diisocyanate [14]. The use of 
diisocyanate to end-cap a hydroxyl-terminated polymer such as the $\mathrm{PB}-\mathrm{OH}$ under investigation was not successful because it is associated with several constraints.

From a chemical point of view. A carbamate function results from the reaction of a diisocyanate with a hydroxyl terminated polymer. This carbamate constitutes a weak site in the propagating species which is easily attacked by $\boldsymbol{\epsilon}$-caprolactam anion.

As illustrated in the reaction scheme in Fig. 3, an undesired side reaction (path B) occurs and favors the formation of homopolyamide and the subsequent release of the initial homohydrogenated polybutadiene. In addition, a carbamate, which is highly sensitive to water (humidity) may readily be hydrolyzed, resulting in chain cleavage, during subsequent polymer processing at high temperatures. In that context, our objective was to avoid reaction B and to favor reaction A which leads to the formation of the desired propagating entity 1 (resulting in PB- $b$-PA6 diblock copolymer) and to obtain a copolymer which is more stable thermally when melt processed at the usual processing conditions.

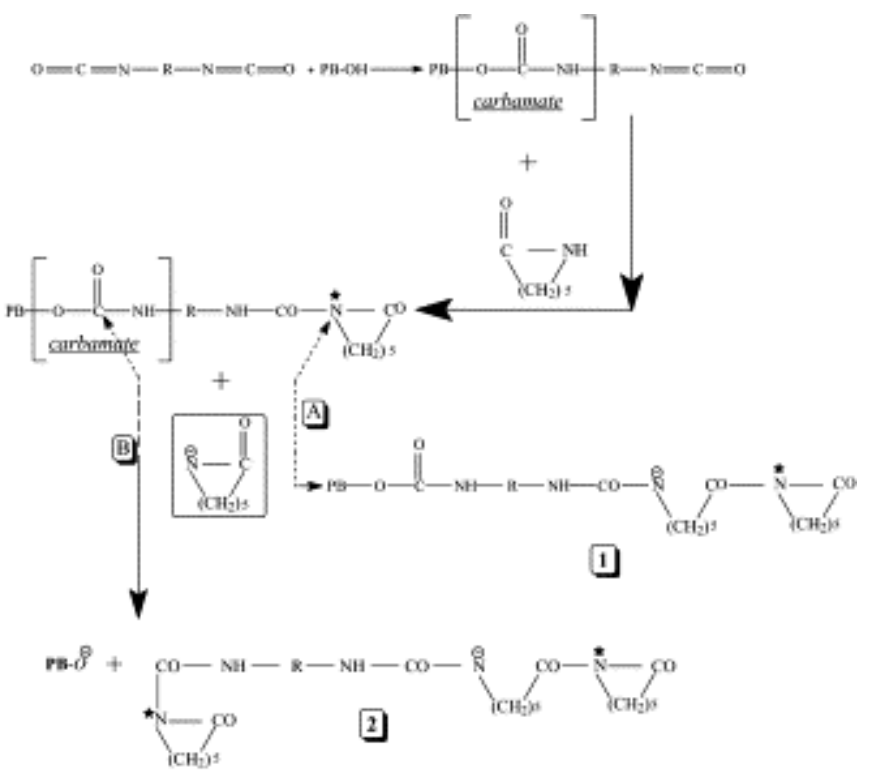

Fig. 3. PB-OH functionalization (with non-protected diisocyanate) and copolymerization reaction scheme showing the propagating species and the side reaction (path B).

From a practical point of view. When polybutadiene is end-capped with diisocyanate, the unreacted excess must be completely eliminated in order to avoid excessive homopolyamide formation during the copolymerization step. This is practically difficult since isocyanate is very sensitive to hydrolysis. Hence, the use of $t$-caprolactam blocked diisocyanate was necessary [15-20]. This approach allows an easy removal of the unreacted isocyanate by methanol successive washing process without any risk of functionality loss via hydrolysis.

Compatibilization ability of HPB-b-PA6 in LDPE/PA 6 blends. Because of their high interfacial tension $(\gamma=10.7 \mathrm{mN} / \mathrm{m})$ and their dissimilar chemical structure, low-density polyethylene and polyamide 6 are highly immiscible [21]. Their blending results in a cheesy material that is mechanically weaker than paper. La Mantia has experimentally confirmed this expected behavior [22]. In the present research the compatibilization ability of the synthesized HPB- $b$-PA6 diblock copolymer in LDPE/PA6 blends has been investigated. Two opposite compositions, i.e. 80/20 and 20/80 wt/wt of LDPE/PA6 were studied. 
As shown in Fig. 4 and Fig. 5 at 80LDPE/20PA6, PA6 phase is dispersed in the form of quasi-spherical particles in LDPE matrix. In the absence of any compatibilizer the average size of these particles is about $2.5 \mu \mathrm{m}$ (Fig. 6). The cryo-fracture surfaces of the unmodified blend observed by SEM presented in Fig. 5(a) reveal clearly that PA6 particles are weakly adhering to the matrix and that their surfaces are smooth. These morphological features are clear evidence and a direct consequence of a lack of adhesion between PA6 particles and LDPE matrix. Upon addition of $10 \mathrm{wt} \%$ of HPB- $b$-PA6 pure diblock copolymer either $\mathrm{C} 2$ or $\mathrm{C} 3$, the phase morphology of the blend is completely modified. In Fig. 4 is illustrated the SEM micrographs of trifluroethanol etched surfaces of the pure and HPB- $b$-PA6 copolymer modified blends. The copolymer activity is unambiguously evidenced in the case of $\mathrm{C} 2$ or $\mathrm{C} 3$ modified blends where a significant reduction of the PA6 particle size is clearly visible. The copolymer C3 causes a PA6 phase size reduction by a factor as high as 13 whereas the addition of copolymer $\mathrm{C} 2$ to the same blend resulted in a reduction by only a factor of five. This differential situation was not expected since C2 and C3 have molecular weight and compositions that are not too different to result in such a discrepancy in terms of interfacial activity. However, when the DSC crystallization exotherms (Fig. 7) of these copolymers are compared to those of the pure HPB and PA6 components substantial differences are depicted. The broader crystallization exotherm exhibited by $\mathrm{C} 2$ copolymer is ascribed to the effect of its higher 1,2 polybutadiene microstructure content; $333 \%$ compared to $12 \%$ in the case of $\mathrm{C} 3$. A hydrogenated 1,2 polybutadiene is less miscible with low-density polyethylene than a hydrogenated 1,4 polybutadiene. The formation of 1,2 microstructure is very sensitive to the polarity of the polymerization medium. The ratio of 1,2 to 1,4 microstructures affects considerably the crystallization behavior of the HPB block. A broad crystallization or melting thermogram is usually obtained when the 1,2 content of the HPB is significant.

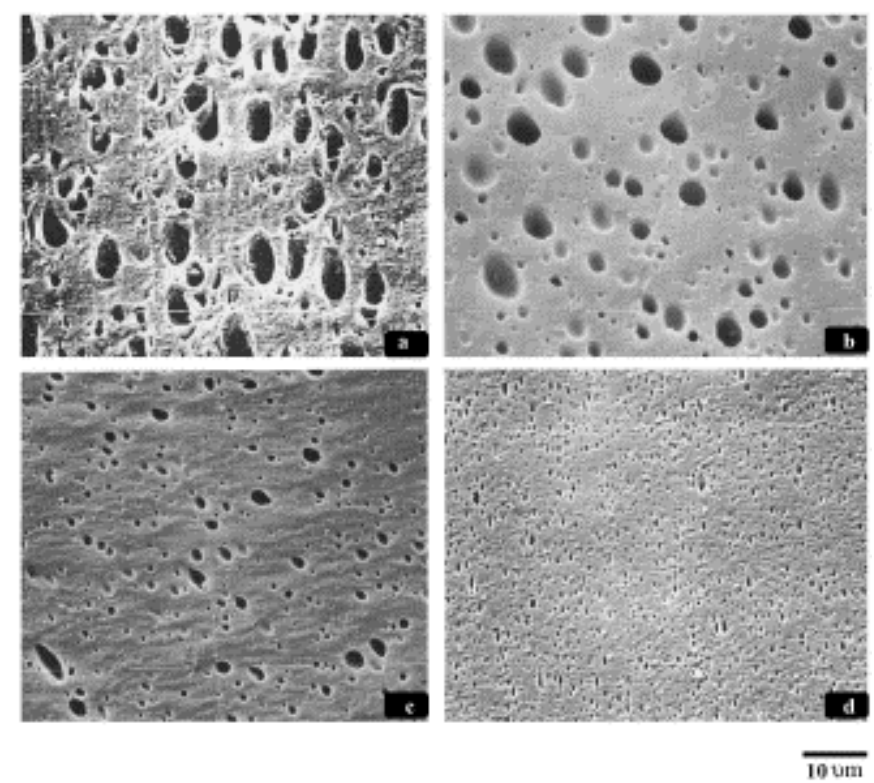

Fig. 4. SEM of cryo-smoothed and TFE etched surfaces of 80LDPE/20PA6: without copolymer (a) and added with 10 wt\% HPB-b-PA6 diblock copolymer: (b) C1, (c) C2 and (d) C3. 

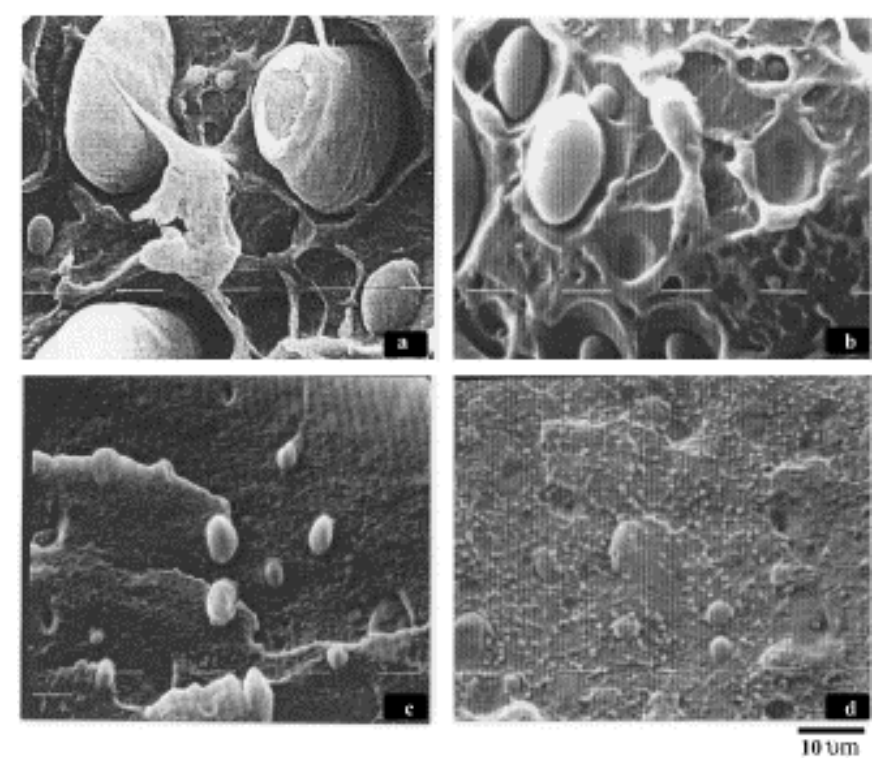

Fig. 5. SEM of cryo-fracture surfaces of 80LDPE/20PA6: without copolymer (a) and added with 10 wt\% HPB-b-PA6 diblock copolymer: (b) C1, (c) C2 and (d) C3.

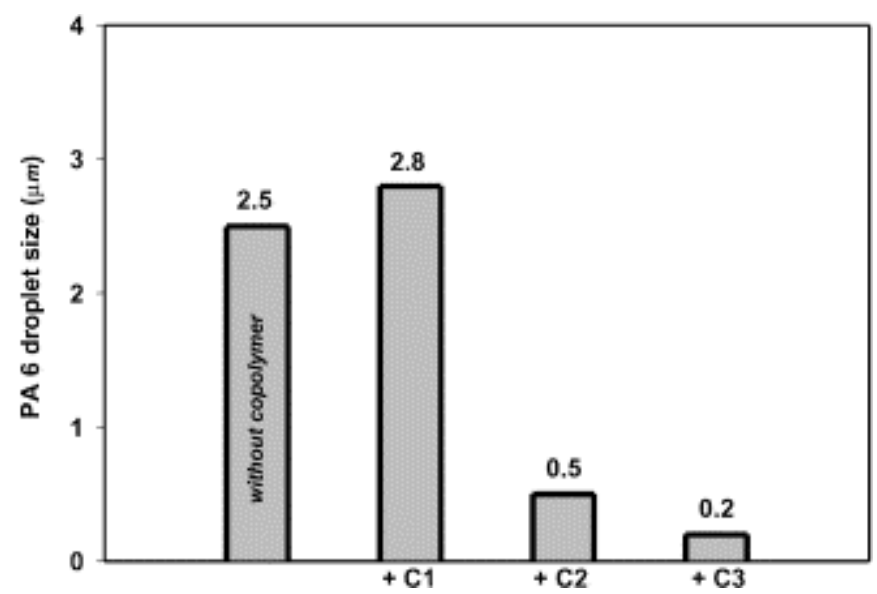

Fig. 6. PA6 particle diameter of HPB-b-PA6 modified 80LDPE/20PA6. 


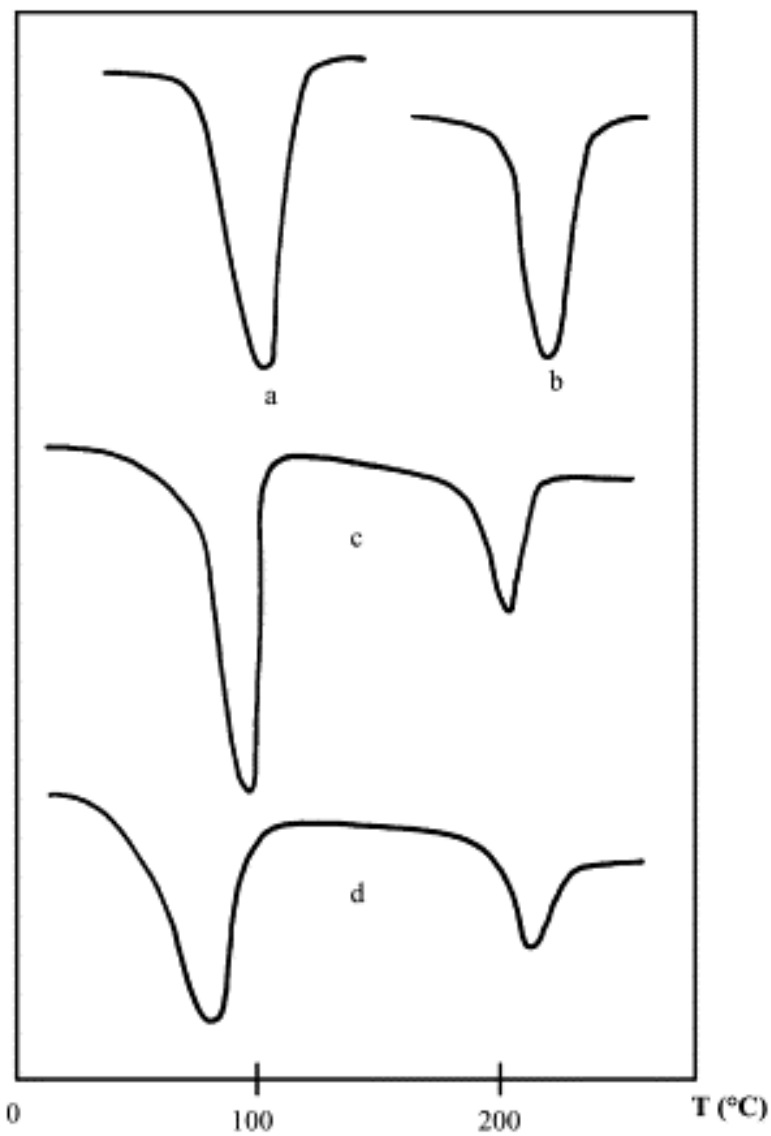

Fig. 7. DSC crystallization exotherms of (a) Hydrogenated polybutadiene, (b) homopolyamide 6 and, (c) C3 and (d) C2 HPB-b-PA6 diblock copolymer.

When copolymer $\mathrm{C} 1$ is used a slight phase growth compared to the unmodified blend is observed (Fig. 6). This low molecular weight copolymer exhibits a low shear viscosity. As shown in Table 2 and Fig. 8, the addition of $5 \mathrm{wt} \%$ of $\mathrm{C} 1$ to pure LDPE results in a viscosity decrease of the latter by a factor of about 6.5. Whereas its addition to PA 6 homopolymer does not really affect the viscosity of the mixture $\mathrm{C} 1$ in PA6. That might judiciously indicate that in the LDPE/PA6 blend $\mathrm{C} 1$ is probably preferentially dispersed in the LDPE matrix. In that case, the viscosity ratio of PA6 to LDPE is modified. Larger PA6 particles are expected in the resulting less viscous ( $\mathrm{C} 1$ in LDPE) matrix. The dependence of dispersed particle size on dispersion to matrix viscosity ratio has already been demonstrated [23]. When the matrix exhibits a much lower viscosity than that of the dispersion, shear is poorly transferred and particle break-up is not optimized, resulting in large particle size.

Table 2. Dynamic viscosity $\left(\eta^{\prime}\right)$ and storage $\left(G^{\prime}\right)$ modulus for unmodified and $C 1, C 2$ and $C 3$ modified LDPE and PA6 at $25 \mathrm{rad} / \mathrm{s}$

\begin{tabular}{ccccc}
\hline & LDPE & LDPE+C1 & LDPEAC2 & LDPEAC3 \\
\hline$\eta^{\prime}(\mathrm{Pas})$ & 842 & 180 & 1080 & 1150 \\
$G^{\prime}(\mathrm{Pa})$ & 29640 & 4513 & 27530 & 28630 \\
& PA6 & PA6+C1 & PA6+C2 & PA6+C3 \\
$\eta^{\prime}(\mathrm{Pa} \mathrm{s})$ & 860 & 654 & 710 & 680 \\
$G^{\prime}(\mathrm{Pa})$ & 8000 & 6524 & 8730 & 8200 \\
\hline
\end{tabular}



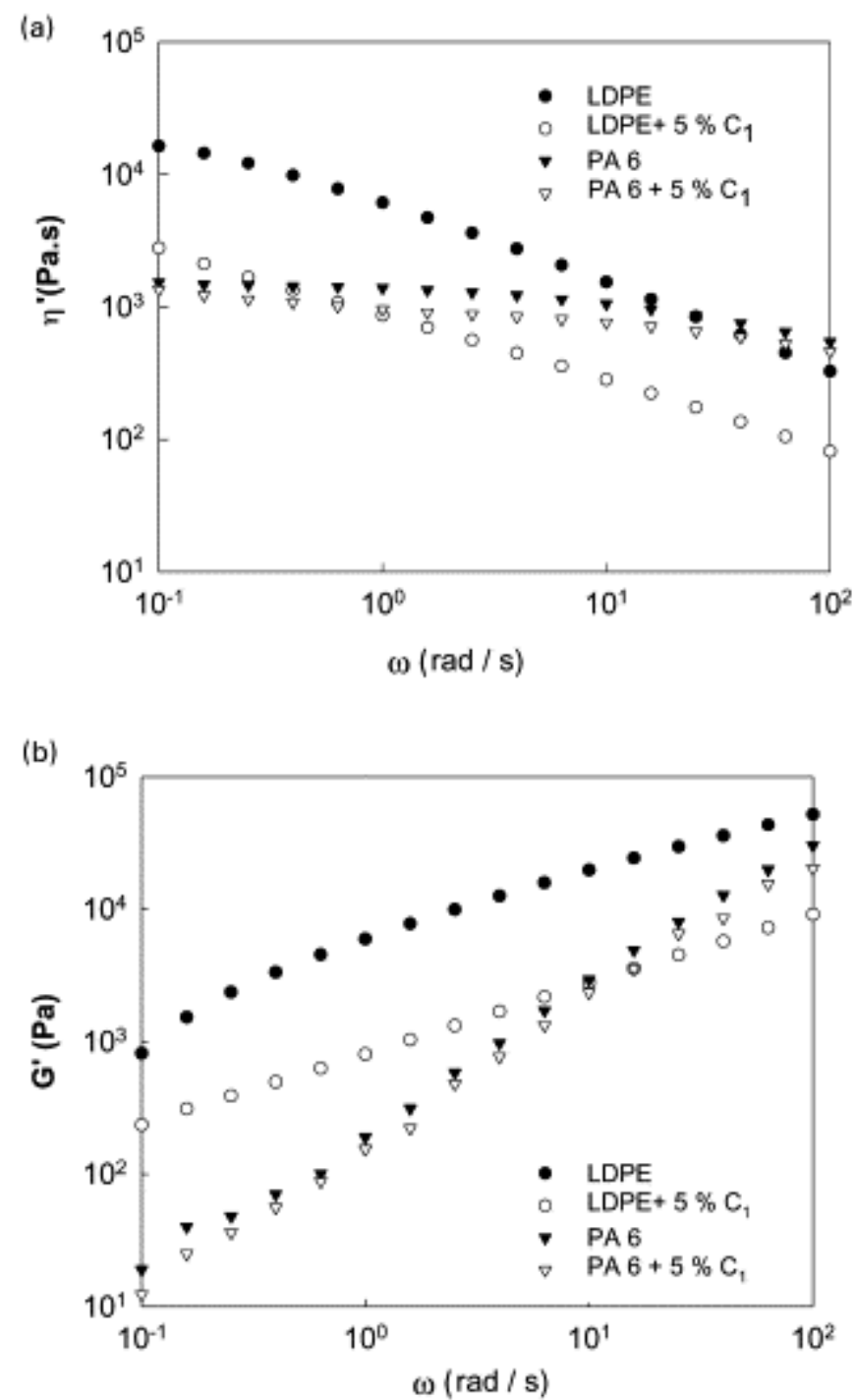

Fig. 8. (a) Dynamic viscosity $\left(\eta^{\prime}\right)$ and (b) storage modulus $\left(G^{\prime}\right)$ versus frequency of unmodified and $5 \mathrm{wt} \% \mathrm{C1}$ modified LDPE and PA6 homopolymers.

SEM micrographs of the cryo-fracture surfaces shown in Fig. 5 reveal that upon addition of the HPB- $b$-PA6 diblock copolymer, PA6 particles become well adhered to LDPE matrix. The effect is more pronounced in the case of $\mathrm{C} 3$ modified blend than in the case of C2 whereas $\mathrm{C} 1$ modified one does not exhibit any adhesion between PA6 and LDPE (Fig. 5(b)).

From the earlier results, it comes out that $\mathrm{C} 3$ copolymer exhibits the most efficient interfacial activity in 80LDPE/20PA6 blends. C1 copolymer, which was found to exhibit no compatibilizing effect, is most probably mainly dispersed in the LDPE phase.

As pointed out in the experimental part, under the mixing conditions used, a co-continuous phase morphology was developed in the 20LDPE/80PA6 blends. However, in the absence of suitable compatibilizer that co-continuous phase morphology was no longer stable upon compression molding at $240{ }^{\circ} \mathrm{C}$ for $3 \mathrm{~min}$. This situation is illustrated in the optical micrographs of Fig. 9(a) and (b). The micrograph (a) was obtained from a microtomed section of a 20LDPE/80PA6 sample quenched in liquid nitrogen just after the milling process;

micrograph (b) was obtained from a 3 min compression molded and water cooled sample. The dispersed morphology so-obtained clearly demonstrates that the co-continuous structure 
induced during mixing evolved as a result of thermal annealing at $240{ }^{\circ} \mathrm{C}$ and upon cessation of shear. The selective extraction of PA6 by using trifluoroethanol constitutes an efficient technique for unambiguously proving the co-continuous nature of these morphologies. As shown in Table 3, after selective and complete PA6 extraction, the liquid nitrogen quenched sample was able to preserve its initial shape without any LDPE loss. However, the compression molded one was totally fragmented (destroyed into suspended LDPE particles) after only $2 \mathrm{~h}$ of PA6 phase extraction.
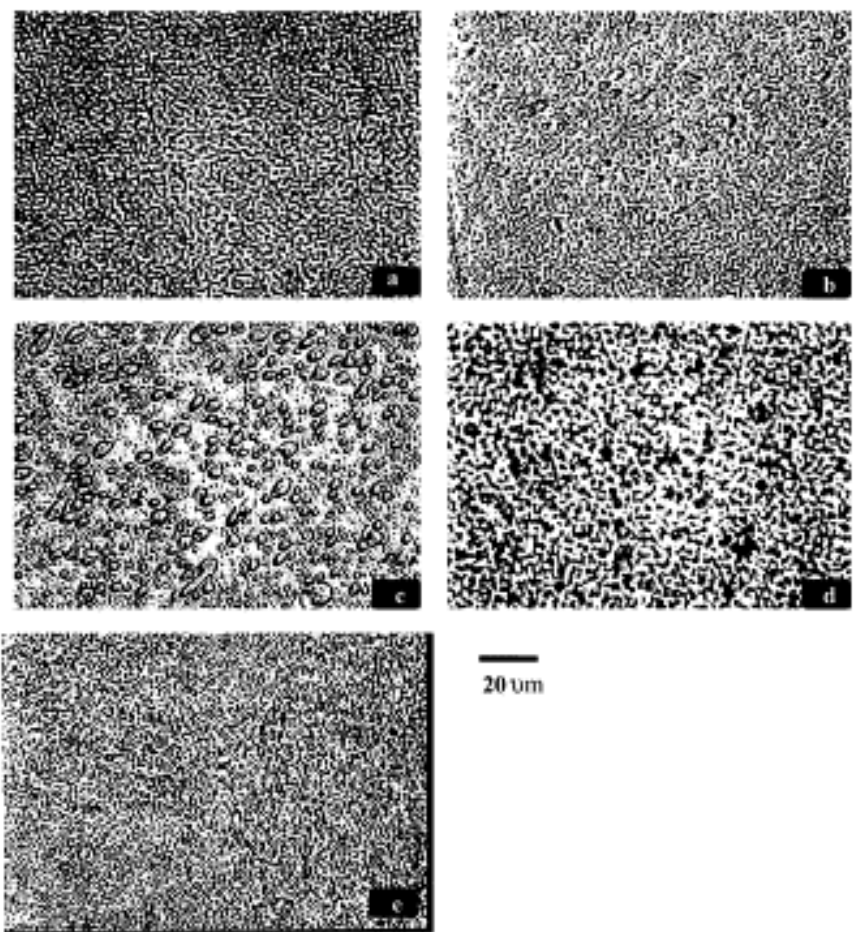

\section{$\overline{200 \mathrm{~m}}$}

Fig. 9. Optical micrographs of 20LDPE/80PA6-without copolymer: (a) quenched sample and (b) molded for 3 min at $240{ }^{\circ} \mathrm{C}$-added with $10 \mathrm{wt} \% \mathrm{HPB}-b-P A 6$ (c) C1, (d) C2, (e) C3.

Table 3. 20LDPE/80PA6+10 wt\% HPB- $b$-PA6 copolymer: results on PA6 phase extraction

\begin{tabular}{|c|c|c|c|c|c|}
\hline Blend & Extraction time (h) & After extraction & $\begin{array}{l}\text { W1\% of LDPE after extrac- } \\
\text { tion }\end{array}$ & Solvent aspect & Conclusion \\
\hline $\begin{array}{l}\text { Wifhout copolymer } \\
\text { quenched }\end{array}$ & 144 & Intact & 98 & Transparent & $\begin{array}{l}\text { Co-continuous inorphal- } \\
\text { ogy }\end{array}$ \\
\hline Without copdymer molded & 5 & Fragmented & - & Cloudy dispersion & Droplet-in-matix \\
\hline$+\mathrm{C} 1$ & 51 & Totally fragmented & - & Cloudy dispersion & Droplet-in-manix \\
\hline$+\mathrm{C}_{2}$ & 144 & Slightly 'dedaminated' & 75 & Few panticles saspended & Semi-contimuous \\
\hline$+\mathrm{Cs}$ & 144 & Intact & 99.2 & Transparent & $\begin{array}{l}\text { Co-continuous monphol- } \\
\text { ogy }\end{array}$ \\
\hline
\end{tabular}

The results of the extraction experiments presented in Table 3 clearly reveal that the blend modified by $\mathrm{C} 1$ is totally fragmented in trifluroethanol which implies a droplet-in-matrix phase morphology. This is in good agreement with the 2D optical micrograph ( Fig. 9(c)) showing visible PA6 particles dispersed in LDPE matrix. The copolymer C1 was thus not able to stabilize the co-continuous phase morphology of the 20LDPE/80PA6 from evolving to droplet-in-matrix phase morphology. A close comparison of micrographs ( Fig. 9(a) and (c)) reveals that the particle size of $\mathrm{C} 1$ modified blend is larger than that of the unmodified blend. This effect is ascribed to the modification of the viscoelastic properties of LDPE induced by the addition of $\mathrm{C} 1$. The extraction of the LDPE phase from accessible outer surfaces of the blend using dichloroethylene revealed that about $75 \mathrm{wt} \%$ of the initial amount of $\mathrm{Cl}$ was 
drawn into the solvent in the form of swollen and dispersed particles. This finding confirms the preferential location of C1 in LDPE Phase.

C2 modified blend although exhibits slight delamination of LDPE phase upon PA6 extraction it was able to preserve its initial shape. The weight of the remaining compact mass reveals that about $25 \%$ of the LDPE phase was sub-included in PA6 major phase. C2 was thus able to stabilize about $75 \%$ of the phase co-continuity. The optical micrograph (Fig. 9(d)) corresponding to $\mathrm{C} 2$ modified blend agrees quite well with the extraction result. It is important to indicate that the $25 \%$ LDPE sub-inclusions are difficult to distinguish in a 2D optical micrograph.

In the case of $\mathrm{C} 3$ modified blend the solvent recovered was quasi-transparent and almost free of insoluble particles. As shown in Table 3 all LDPE phase including the copolymer that remained attached to the interface remained in a continuous intact piece after the extraction of PA6 phase. The optical micrograph presented in Fig. 9(e) shows a very fine co-continuous phase morphology of $\mathrm{C} 3$ modified blend in good agreement with the extraction result. The copolymer C3 was thus able to totally stabilize the developed co-continuous phase morphology.

Tensile strength $\sigma_{\mathrm{b}}$ and elongation at break $\boldsymbol{t}_{\mathrm{b}}$ of the modified and unmodified 80LDPE/20PA6 blends are shown in Fig. 10(a) and (b), respectively. The copolymer C3 imparts to the blend the highest elongation at break and tensile strength compared to $\mathrm{C} 2$. That differential behavior might also originate from a lack of miscibility of the HPB block of copolymer C2 with LDPE phase. No significant improvement in tensile strength is observed in the case of $\mathrm{C} 1$ modified blend. The enhancement of elongation at break by a factor of 1.6 results from a plasticizing effect of the matrix. In Fig. 11(a) and (b) are shown the tensile strength and elongation at break of the unmodified and modified 20LDPE/80PA6 blends. As in the case of LDPE rich blends (droplet-in-matrix phase morphology), C3 and C2 modified blends also exhibit the highest tensile strength and elongation at break compared to the unmodified or $\mathrm{C} 1$ modified one. This is a good indication of their quantitative location at LDPE/PA6 interface resulting in significant improvement of the interfacial adhesion. 
Published in: Polymer (2002), vol. 49, iss. 19, pp. 5347-5354

Status: Postprint (Author's version)
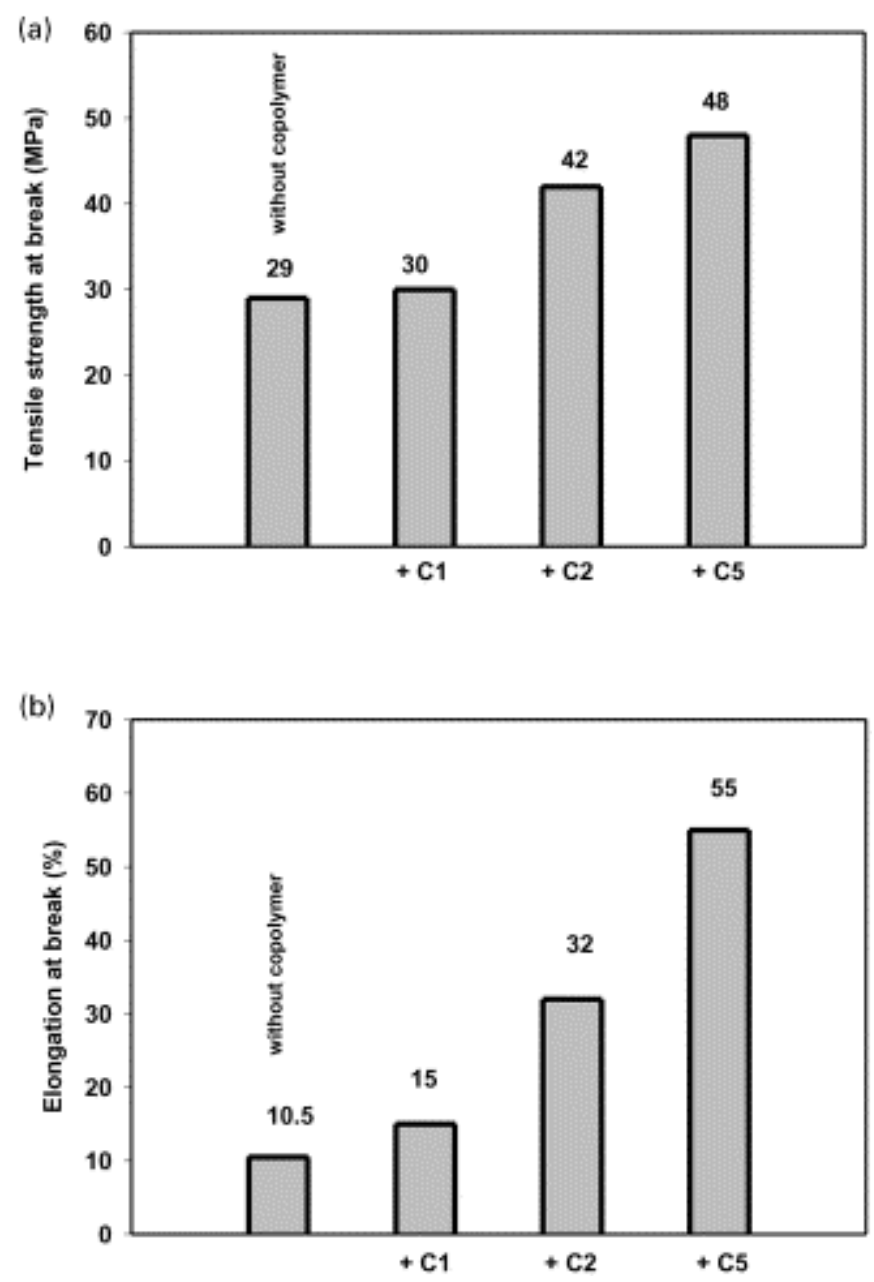

Fig. 10. 80LDPE/20PA6+10 wt\% HPB-b-PA6 diblock copolymer: (a) Tensile strength and (b) Elongation at break. 

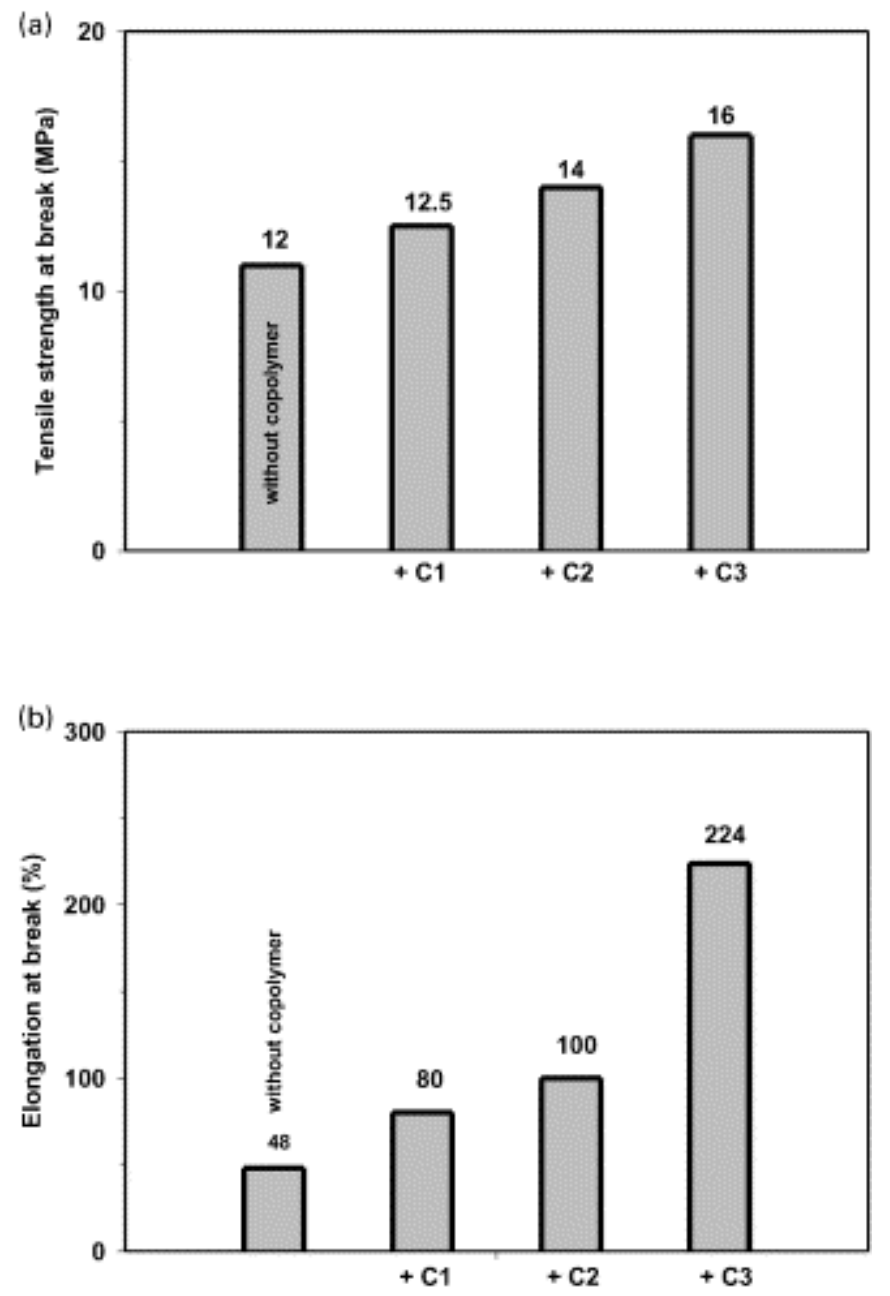

Fig. 11. 20LDPE/80PA6+10wt\% HPB-b-PA6 diblock copolymer: (a) Tensile strength at break $\left(\sigma_{b}\right)$, and (b) Elongation at break $\left(\mathbf{E}_{b}\right)$.

\section{Conclusion}

Although the synthesis procedure may appear to be relatively long, it allowed us for the first time to synthesize a pure hydrogenated polybutadiene- $b$-polyamide 6 diblock copolymer which is thermally stable at the processing conditions of LDPE/PA6 blends. When further optimized, this copolymer might be a very good candidate for the substitution of commonly used reactive compatibilizing agents. This approach of using pre-made HPB- $b$-PA6 pure diblock copolymer of well-controlled molecular structure will open a new area of investigation in the compatibilization of polyethylene polyamide blends.

Based on both the droplet-in-matrix and the co-continuous phase morphology developed in the blends studied, it was demonstrated that a HPB- $b$-PA6 diblock copolymer of 87,000 molar mass and $24 \mathrm{wt} \%$ of PA6 exhibits an excellent compatibilization ability in LDPE/PA6 blends. A very fine dispersion of PA6 particles in LDPE matrix was obtained. The copolymer was able to stabilize the co-continuous phase morphology developed in 20LDPE/80PA6 blend.

It is also evidenced that a low molecular weight $(12,500)$ HPB-b-PA6 diblock containing $20 \mathrm{wt} \%$ of PA6 exhibit no emulsifying activity. This deficiency was ascribed to its preferential location in LDPE phase. 


\section{Acknowledgements}

The present research work was funded by Euro BRITE and Alcatel-Alsthom, France. The authors would like to thank Prof. Ph. Teyssié, R. Gadessaud, J.Y. Barraud and J.F. Fauvarque for their advise and support.

\section{References}

[1] Triacca VJ, Ziaee S, Barlow JW, Keskkula H, Paul DR. Polymer 1991;32:1401.

[2] Takeda Y, Paul DR. Polymer 1991;32:2771.

[3] Lu M, Keskkula H, Paul DR. Polym Engng Sci 1994;34:33.

[4] Duvall J, Sellitti C, Topolkaraev V, Hiltner A, Baer E, Myers C. Polymer 1994;35:3948.

[5] Marechal Ph. PhD Thesis. Université Catholique de Louvain, Belgium; 1993. [6] De

Roover B. PhD Thesis. Université Catholique de Louvain, Belgium; 1994.

[7] Coran AY, Patel R, Williams-Head D. Rubber Chem Technol 1985; 58:1014.

[8] Baker RE, Saleem M. Polymer 1987;28:2057.

[9] Angola JC, Fujita Y, Sakai T, Inoue T. J Polym Sci, Polym Phys Ed 1988;26:807.

[10] Borggreve RJM, Gaymans RJ. Polymer 1989;30:63.

[11] Fayt R, Jérôme R, Teyssié Ph. J Polym Phys Ed 1982;20:2209.

[12] Falk JC. J Polym Sci A-1 1971;9:2617.

[13] Petit D, Jérôme R, Teyssié Ph. J Polym Sci: Polym Chem Ed 1979;17: 2903.

[14] Petit D. PhD Thesis. Université of Liège, Belgium; 1975.

[15] Wiley PF. J Am Chem Soc 1949;71:1310.

[16] Wiley PF. J Am Chem Soc 1949;71:3741.

[17] Koning CE, Van Geenen AA, Roerdink E, Fayt R, Teyssié Ph. Dutch Application, 8801914; 1988.

[18] Koning CE, Van Geenen AA, Roerdink E, Fayt R, Teyssié Ph. European Application, 89201978.7; 1989.

[19] Koning CE, Van Geenen AA, Vondervoort EWM, Fayt R, Teyssié Ph. Dutch Application, 8900001; 1989.

[20] Koning CE, Van Geenen AA, Vondervoort EWM, Fayt R, Teyssié Ph. European Application, 89203325.9; 1989.

[21] Brandrup J, Immergut EH. Polymer handbook, 3rd ed. New York: Wiley Interscience; 1989.

[22] La Mantia FP, Valenza A, Acierno D. Colloid Polym Sci 1985;263: 726.

[23] Favis BD, Chalifoux JP. Polym Engng Sci 1987;27:1591. 